\title{
Investigation of $F J 194940.1$ gene alternative splicing in colon cancer and its association with clinicopathological parameters
}

\author{
MALWINA BARTCZAK-TOMCZYK, ALEKSANDRA SAŁAGACKA, MAREK MIROWSKI and EWA BALCERCZAK \\ Laboratory of Molecular Biology and Pharmacogenomics, Department of Pharmaceutical Biochemistry, \\ Medical University of Lodz, Lodz 90-151, Poland
}

Received September 2, 2011; Accepted October 25, 2011

DOI: $10.3892 /$ etm.2011.378

\begin{abstract}
Colorectal cancer (CRC) is one of the most frequent neoplasms and is responsible for the second highest mortality rate of all cancers in the more developed regions of the world. The molecular mechanisms of CRC are relatively well characterized and are correlated to the accumulation of genetic mutations and certain patterns of gene expression/overexpression. There are a number of possible molecular factors involved in CRC progression in the aforementioned pathways, which are as yet not well described. One of these factors appears to be the gene $F J 194940.1$, previously termed P65. FJ 194940.1 consists of 6 exons and probably undergoes alternative splicing in malignant tissues. In this study, tissue samples from 102 patients with colon cancer were investigated to confirm alternative splicing and to correlate results obtained with clinicopathological parameters. A total of 18 splice variants, which arise from various combinations of 4 exons (II, III, IV and V) and exon-exon junctions between exons 1 and 2 (I/II); 2 and 3 (II/III); 3 and 4 (III/IV), as well as 4 and 5 (IV/V), were found. For statistical analysis the full length transcript was divided into parts A and B. Part A consisted of exons II and III, as well as I/II and II/III exon-exon junctions, whereas part B comprised exons IV and $\mathrm{V}$, as well as III/IV and IV/V exon-exon junctions. The expression of part B of the FJ 194940.1 gene transcript is correlated with welldifferentiated (G1) and moderately differentiated cases (G2). Lymphocytic tumor infiltration, a good prognostic factor in CRC, was significantly correlated to the presence of all elements in part A of the FJ 194940.1 gene transcript. Patients who had all elements in part $\mathrm{A}$ of the transcript survived for a shorter
\end{abstract}

Correspondence to: Dr Ewa Balcerczak, Laboratory of Molecular Biology and Pharmacogenomics, Department of Pharmaceutical Biochemistry, Medical University of Lodz, 1 Muszynskiego Street, Lodz 90-151, Poland

E-mail: ewa.balcerczak@umed.lodz.pl

Key words: FJ 194940.1, expression, alternative splicing, transcript, colon cancer duration. Investigation of the FJ 194940.1 gene revealed that the gene had undergone alternative splicing. However, the role of its transcripts and potential proteins should be examined in detail.

\section{Introduction}

Colorectal cancer (CRC) is a serious medical problem. It is one of the most frequently occurring neoplasms and is responsible for the second highest mortality rate of all cancers in the more developed regions of the world. Approximately 220,000 new cases of CRC are diagnosed each year in the USA and Northwestern Europe. The genetic alterations in CRC progression are determined by one of three pathways: microsatellite instability (MSI), chromosome instability (CIN) or CpG island methylator phenotype (CIMP). The progression of CRC is correlated to a number of characteristic mutations in genes $A P C, K-R A S, D C C, P 53$, transforming growth factor- $\beta / S M A D$, or epigenetic changes. There are a number of other possible molecular factors involved in CRC progression, one of which appears to be the gene FJ 194940.1, previously termed P65.

Previous investigation of the gene encoding tumorassociated protein (P65) suggested that it is a molecule that is newly associated with carcinogenesis. Its clinical characterization as a potential marker of cancer development and progression has been reported in many tumors (1-3). Cloning of the complementary DNA (cDNA) encoding this protein revealed that the sequence corresponds to the 226 24025-226 27543 region of chromosome 1 . The gene for this transcript, hereafter termed $F J 194940.1$, consists of 6 exons and probably undergoes differential splicing in malignant tissues. Moreover, integration of an additional copy of the rv_001141 provirus in this region of chromosome 1 was detected. It is possible that this integration leads to the rearranged transcription of FJ 194940.1 (4).

The aim of this study was to assess the presence of the expression of each potential exon and exon-exon junction in the FJ 194940.1 gene transcript. Thereafter, each particular FJ 194940.1 transcript is compared with certain histological parameters and grading, as well as clinical staging of the neoplasms to assess the potential role of the gene transcript as a prognostic marker for CRC. Finally, a comparison of a 
particular FJ 194940.1 transcript with survival time was carried out.

\section{Materials and methods}

Materials. Tissue specimens of colorectal cancer were obtained from the Oncological Centre of Lodz, Poland. CRC was diagnosed by histopathological examination using established clinical criteria (TNM classification by Jass with latest revision Cancer Staging Manual by AJCC, 1997) at the Department of Pathology, Medical University of Lodz, Poland. Tissue samples from 102 patients (55 males and 47 females) were frozen immediately following surgery in liquid nitrogen and stored at $-80^{\circ} \mathrm{C}$ until further examination.

All experiments were carried out with the approval of the local Ethics Committee (No. RNN/214/00). Informed consent was obtained from all 102 patients included in the study.

RNA isolation. Total RNA isolation was performed with a Total RNA Prep Plus Minicolumn Kit (A\&A Biotechnology, Poland) as per the manufacturer's instructions. This method is based on RNA isolation methodology developed previously by Chomczynski and Sacchi, 1987 (5). The estimated degree of contamination of the RNA received was determined using spectrophotometric analysis. The isolated RNA had an A260:280 ratio of 1.6-1.8. Purified RNA samples were stored at $-80^{\circ} \mathrm{C}$ until further use.

Reverse transcriptase polymerase chain reaction (RT-PCR). RT-PCR reaction was carried out using Enhanced Avian HS RT-PCR Kit (Sigma, St. Louis, MO, USA) according to the manufacturer's instructions. The cDNA was used immediately or otherwise stored at $-20^{\circ} \mathrm{C}$. The presence of cDNA in each sample was confirmed using PCR with primers complementary to the $\beta$-actin gene. Only samples with a product of this housekeeping gene were used in subsequent tests.

In the second stage, PCR was performed with particular primers that are specific for potential exons and exon junctions of the FJ 194940.1 gene. The primers were designed using Primer3 software (http://biotools.umassmed.edu/bioapps/ primer3_www.cgi). Amplification conditions were established by gradient PCR. Amplification of particular exons and exon junctions were carried out using $0.7 \mu \mathrm{l} 0.5 \mu \mathrm{M}$, each forward and reverse primers, and $2.5 \mu \mathrm{l}$ cDNA as a template. The PCR mixture also contained $0.4 \mu 110 \mathrm{mM}$ mix deoxyribonucleotide triphosphates (dNTPs), $0.2 \mathrm{ml} 0.5$ units of Taq polymerase, $2 \mu \mathrm{l} 10 \mathrm{X}$ reaction buffer. Parallel negative control (without cDNA) was amplified. PCR products were detected in $2 \%$ agarose gel electrophoresis.

Primer sequences and the size of the expected PCR products are shown in Table I.

Statistical analysis. For statistical analysis, the transcript was divided into two parts: A and B. Part A consisted of exons II and III, as well as I/II and II/III exon-exon junctions, whereas part B comprised exons IV and V, as well as III/IV and IV/V exon-exon junctions. Statistical analyses were performed using STATISTICA 8.0 (StatSoft, Inc., 1984-2008) on the basis of the Chi-square test, Chi-square test with Yates' correction and V2 test. $\mathrm{P}<0.05$ was considered to indicate statistical significance. Time-to-death distribution for survival in the whole population of the 102 patients was estimated using the Kaplan-Meier method. The log-rank test was used to test for differences in time-to-death distribution.

\section{Results}

Alternative transcripts. A total of 18 splice variants were identified, which arose from various combinations of 4 exons (II, III, IV and V) and exon-exon junctions between exons 1 and 2 (I/II), 2 and 3 (II/III), 3 and 4 (III/IV), as well as 4 and 5 (IV/V) (Table II). In the majority of cases, a transcript consisting of all the previously mentioned elements was found. In all 102 samples, PCR products with primers set for exon $\mathrm{V}$ and I/II exon junctions were present. This result indicates that exon $\mathrm{V}$ is common to all mRNA transcripts for the FJ 194940.1 gene. The remaining primer set products yielded inconsistent results. Of note, in 17 cases, PCR products with primer sets for exons II and III were found. However, PCR products with a primer set for the junction of II/III exons were not detected. A similar situation exists in cases of other primers complementary to the exon-exon boundaries and separate exons. This suggests the presence of additional sequences in this area of the FJ 194940.1 transcript.

FJ 194940.1 gene expression is correlated with certain clinico-histological features. Statistical analysis was carried out with reference to the whole FJ 194940.1 transcript and to particular exons and exon-exon junctions. In addition, the whole transcript was divided into parts A and B. Part A consisted of exons II and III, as well as I/II and II/III exon-exon junctions, whereas part $\mathrm{B}$ comprised exons IV and $\mathrm{V}$, as well as III/IV and IV/V exon-exon junctions.

The patients in this study included 55 females and 47 males. The majority of patients had negative familial history. No statistically significant correlation was found between gender and family history and expression of the whole transcript, or parts A or B.

Tumors in the examined group were localized mainly in the colon. No statistically significant correlation was noted between localization of tumor and expression of the whole transcript, or part A or B.

Examined cases were classified as either tubular or mucinosum. There was no statistically significant correlation between histological type and expression of the whole FJ 194940.1 gene transcript, or part A or B. However, there was a tendency towards a more frequent presence of part $\mathrm{A}$ $(\mathrm{p}=0.0894)$ in tubular-type cancer.

Two analyses were conducted between histological grade and splice variants. In the first approach, cases classified as G1 were compared with cases classified as G2 or G3. In the second approach, cases classified as G1 or G2 were compared with cases classified as G3. Expression of part B of the FJ 194940.1 gene transcript is correlated with well-differentiated (G1) and moderately differentiated (G2) cases $(\mathrm{p}=0.0000)$.

To assess the clinical utility of FJ 194940.1 splice variants, determination of these results was compared with various clinicopathological parameters, including depth of tumor invasion (T), lymph node metastases $(\mathrm{N})$ and distant metastases (M). No statistically significant associations were noted 
Table I. Primer sequences specific for potential exons and exon junctions of the FJ 194940.1 gene and expected product size.

\begin{tabular}{lllc}
\hline Exon & \multicolumn{2}{c}{ Primer sequence } & $\begin{array}{c}\text { Expected } \\
\text { amplicon length } \\
\text { (bp) }\end{array}$ \\
\cline { 2 - 4 } & \multicolumn{1}{c}{ Forward primer } & \multicolumn{1}{c}{ Reverse primer } & 153 \\
I/II & TGGTGTCCTATGGAATGCAG & CAGTTCTTCTGGCCCATCCT & 103 \\
II & CACTAGAAAACCCTATGACTTTCACA & GGACAGTTACTTGCAGTTCTTCTG & 155 \\
II/III & TGTGAAACAAGCAGTGCAAC & GCTTGTTGGTCAGCCTTCTG & 101 \\
III & TTTTCCATGTTGATGCTCA & CGCCTGAGTCTGCAGTAAT & 218 \\
III/IV & TGCTCATGCATCTCTGCTTT & TTTGAAATGGGAGCCACTGT & 189 \\
IV & CAGTGGCTCCCATTTCAAAG & TCAAACAGGTGATCGTTCCA & 155 \\
IV/V & CAGCTGGCCTAATCGAAAGA & TCCAGCATTTCAGCAAGAGA & 94 \\
V & CTCTCTTGCTGAAATGCTGG & GGCCCAGGCTTTAAACTATA & \\
\hline
\end{tabular}

between TNM classification and the expression of the whole FJ 194940.1 gene transcript, or part A or B.

Lymphocytic tumor infiltration, a favorable prognostic factor in CRC, was significantly correlated with the presence of all elements in part A of the FJ 194940.1 gene transcript $(\mathrm{p}=0.0477)$.

Venous invasion was observed in the majority of patients. There was no statistically significant association between venous invasion and expression of the whole FJ 194940.1 gene transcript, or part A or B.

The results for the expression of the FJ 194940.1 gene transcript and clinicopathological parameters are shown in Table III.

FJ19490.1 gene and survival time. Statistical analysis between each separate exon and exon-exon junction and survival time was carried out. Notably, if we analyzed the survival time dependent on the presence or absence of separate exons and boundaries, only the presence of the junction between exons II/III was likely to be correlated with a longer survival time. This correlation was statistically significant $(\mathrm{p}=0.01972)$.

No statistically significant difference was observed in survival time when comparing patients with the presence and absence of the whole transcript, despite a marked tendency for longer survival of patients without at least one element in the whole transcript $(\mathrm{p}=0.0690)($ Fig. 1A).

A statistically significant difference was found in survival time when comparing patients with presence or absence of part A of the FJ 194940.1 gene transcript. Patients who had all elements in part $\mathrm{A}$ of the transcript survived for a shorter duration ( $\mathrm{p}=0.0051$ ) (Fig. 1B).

There was no statistically significant difference in survival time when comparing patients with the presence or absence of part B of the FJ 194940.1 gene transcript (Fig. 1C).

\section{Discussion}

Splicing, the production of more than one transcript from one gene, appears to affect over $50 \%$ of human genes. Numerous cancer-associated genes, including $C D 44$ and $W T 1$, are alternatively spliced. Variation of the splicing process may occur during tumor progression and play a significant role in tumori-
Table II. Combination and frequency (A) of 18 possible splice variants.

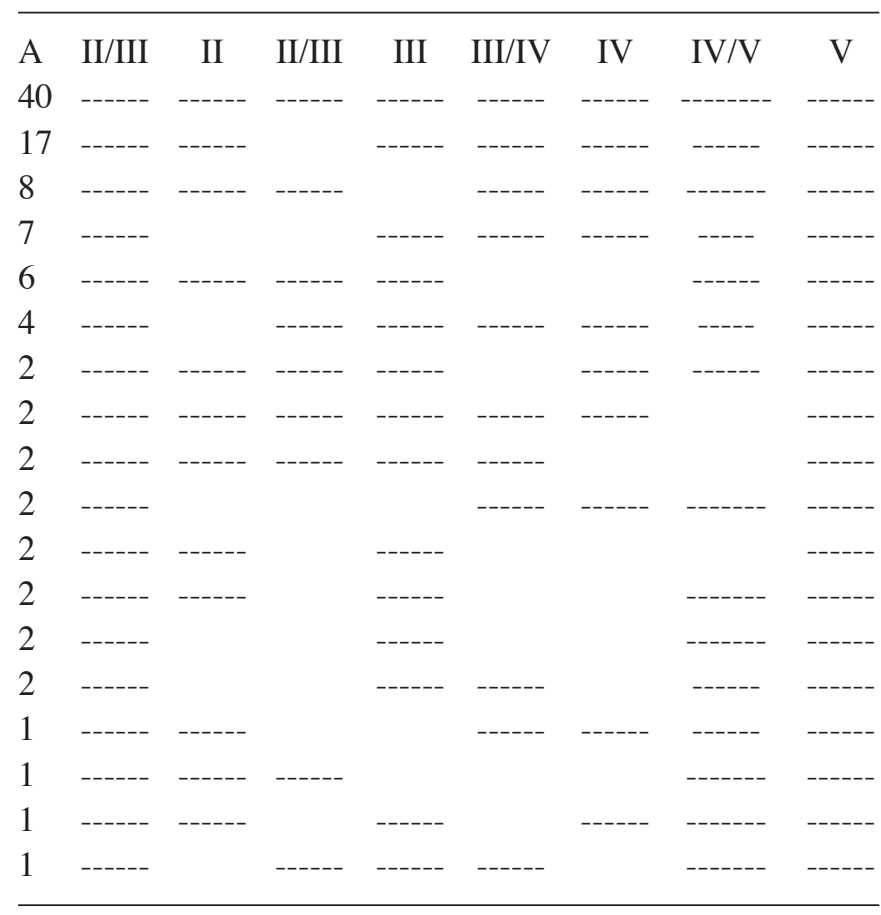

genesis (6-12). Furthermore, alternatively spliced transcripts, which are found mainly in tumor tissues, may be extremely useful as cancer markers and serve as drug targets. In addition, clarification and understanding of the changes in splicing, as well as the characterization of various isoforms, may improve our knowledge of malignant transformation.

Probably the best characterized genes that exist in several isoforms and play a role in carcinogenesis are $C D 44$ and the Wilms' tumor gene, WT1. CD44 standard and CD44 variants are expressed in a number of cancer cell types and correlate with the progression and prognosis of certain malignant tumors. For example, CD44v6 expression was positively connected with advanced gastric cancer stage, and patients with this isoform have lower, 3- and 5-year survival times (10). In their study, Aaltomaa et al demonstrated that 
Table III. Comparison of the expression of a particular FJ 194940.1 transcript with certain clinicopathological parameters.

\begin{tabular}{|c|c|c|c|c|c|c|c|c|c|}
\hline \multirow[t]{2}{*}{ Parameters } & \multicolumn{3}{|c|}{$\begin{array}{l}\text { Expression of the whole } \\
\text { transcript FJ 194940.1 gene }\end{array}$} & \multicolumn{3}{|c|}{$\begin{array}{c}\text { Expression of part A } \\
\text { transcript FJ 194940.1 gene }\end{array}$} & \multicolumn{3}{|c|}{$\begin{array}{c}\text { Expression of part B } \\
\text { transcript FJ 194940.1 gene }\end{array}$} \\
\hline & - & + & p-value & - & + & $\mathrm{p}$-value & - & + & p-value \\
\hline \multicolumn{10}{|l|}{ Gender } \\
\hline Female & 34 & 21 & $0.8170^{\mathrm{a}}$ & 28 & 27 & $0.6797^{\mathrm{a}}$ & 13 & 42 & $0.7762^{\mathrm{a}}$ \\
\hline Male & 28 & 19 & & 22 & 25 & & 10 & 37 & \\
\hline \multicolumn{10}{|l|}{ Family history } \\
\hline Negative & 55 & 35 & $0.8969^{b}$ & 45 & 45 & $0.5894^{\mathrm{c}}$ & 19 & 71 & $0.5592^{\mathrm{b}}$ \\
\hline Positive & 7 & 5 & & 5 & 7 & & 4 & 8 & \\
\hline \multicolumn{10}{|c|}{ Tumor localization } \\
\hline Rectum & 20 & 15 & $0.5236^{\mathrm{a}}$ & 16 & 19 & $0.5790^{\mathrm{a}}$ & 6 & 29 & $0.3283^{c}$ \\
\hline Colon & 42 & 24 & & 34 & 32 & & 17 & 49 & \\
\hline \multicolumn{10}{|c|}{ Histological type } \\
\hline Tubular & 51 & 36 & & 41 & 46 & $0.3594^{\mathrm{c}}$ & 19 & 68 & $0.9373^{\mathrm{b}}$ \\
\hline Mucinosum & 11 & 4 & $0.2835^{\mathrm{c}}$ & 9 & 6 & & 4 & 11 & \\
\hline \multicolumn{10}{|c|}{ Histological grade } \\
\hline $\mathrm{G} 1$ or $\mathrm{G} 2$ & 41 & 29 & $0.3826^{\mathrm{a}}$ & 38 & 32 & $0.1487^{\mathrm{a}}$ & 8 & 62 & $0.0000^{\mathrm{c}}$ \\
\hline G3 & 21 & 10 & & 12 & 19 & & 15 & 16 & \\
\hline \multicolumn{10}{|c|}{ Histological grade } \\
\hline $\mathrm{G} 1$ & 9 & 1 & & 8 & 2 & $0.0894^{\mathrm{b}}$ & 2 & 8 & $0.8595^{b}$ \\
\hline $\mathrm{G} 2$ or $\mathrm{G} 3$ & 53 & 38 & $0.1061^{\mathrm{b}}$ & 42 & 49 & & 21 & 70 & \\
\hline \multicolumn{10}{|l|}{ Invasion of the } \\
\hline \multicolumn{10}{|l|}{ intestinal wall } \\
\hline pT1 or pT2 & 18 & 11 & $0.8273^{\mathrm{a}}$ & 14 & 15 & $0.9757^{\mathrm{a}}$ & 6 & 23 & $0.7526^{c}$ \\
\hline pT3 or pT4 & 43 & 29 & & 35 & 37 & & 17 & 55 & \\
\hline \multicolumn{10}{|c|}{ Node involvement } \\
\hline pN0 & 36 & 24 & $0.9586^{\mathrm{a}}$ & 30 & 30 & $0.7996^{\mathrm{a}}$ & 12 & 48 & $0.4676^{\mathrm{c}}$ \\
\hline $\mathrm{pN} 1$ or $\mathrm{pN} 2$ & 23 & 15 & & 18 & 20 & & 10 & 28 & \\
\hline \multicolumn{10}{|c|}{ Distant metastasis } \\
\hline pM0 & 53 & 30 & $0.1864^{c}$ & 44 & 39 & $0.0934^{\mathrm{c}}$ & 19 & 64 & $0.8956^{\mathrm{b}}$ \\
\hline pM1 & 9 & 10 & & 6 & 13 & & 4 & 15 & \\
\hline \multicolumn{10}{|l|}{ TNM stage } \\
\hline I or II & 35 & 22 & $0.8854^{\mathrm{a}}$ & 29 & 28 & $0.6728^{a}$ & 12 & 45 & $0.6840^{\mathrm{a}}$ \\
\hline III or IV & 27 & 18 & & 21 & 24 & & 11 & 34 & \\
\hline \multicolumn{10}{|l|}{ Lymphocytic } \\
\hline \multicolumn{10}{|l|}{ infiltration } \\
\hline Absent & 33 & 25 & $0.4036^{\mathrm{a}}$ & 28 & 30 & $0.7742^{\mathrm{a}}$ & 12 & 46 & $0.7585^{\mathrm{c}}$ \\
\hline Present & 28 & 15 & & 22 & 21 & & 10 & 33 & \\
\hline \multicolumn{10}{|c|}{ Venous invasion } \\
\hline Absent & 28 & 13 & $0.2029^{a}$ & 25 & 16 & $0.0477^{\mathrm{a}}$ & 8 & 33 & $0.5494^{c}$ \\
\hline Present & 34 & 27 & & 25 & 36 & & 15 & 46 & \\
\hline
\end{tabular}

+, presence of all elements; -, absence at least one element; ${ }^{\mathrm{a} C h i}$-square test; ${ }^{\mathrm{b}} \mathrm{Chi}$-square test with Yates' correction; 'test V2.

the down-regulation of CD44 and its CD44v6 isoform was correlated with tumor malignancy and unfavorable prognosis in prostate cancer (11). The expression of CD44 isoforms has also been evaluated in breast cancer. The presence of CD44v3 significantly correlated with the presence of lymph nodes metastases (13). Thus, CD44 variants may be useful as diagnostic or prognostic markers in certain human malignant diseases. However, data are conflicting and further studies are required in order to establish the prognostic value of CD44 and its variant isoforms. This observation is a good example 
A

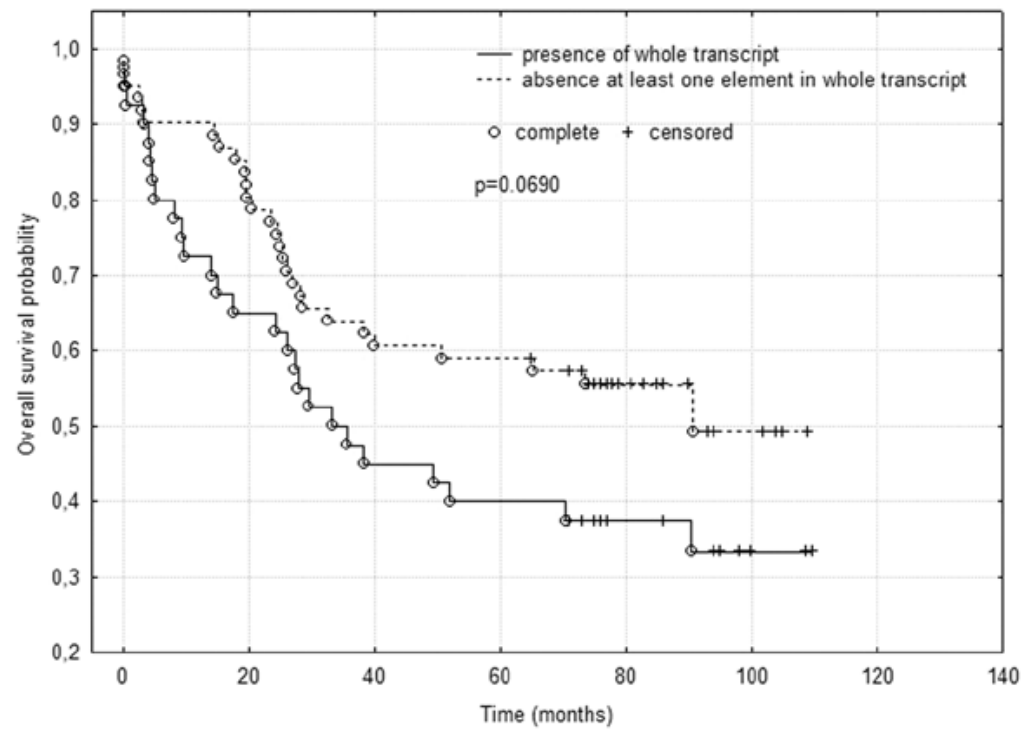

B

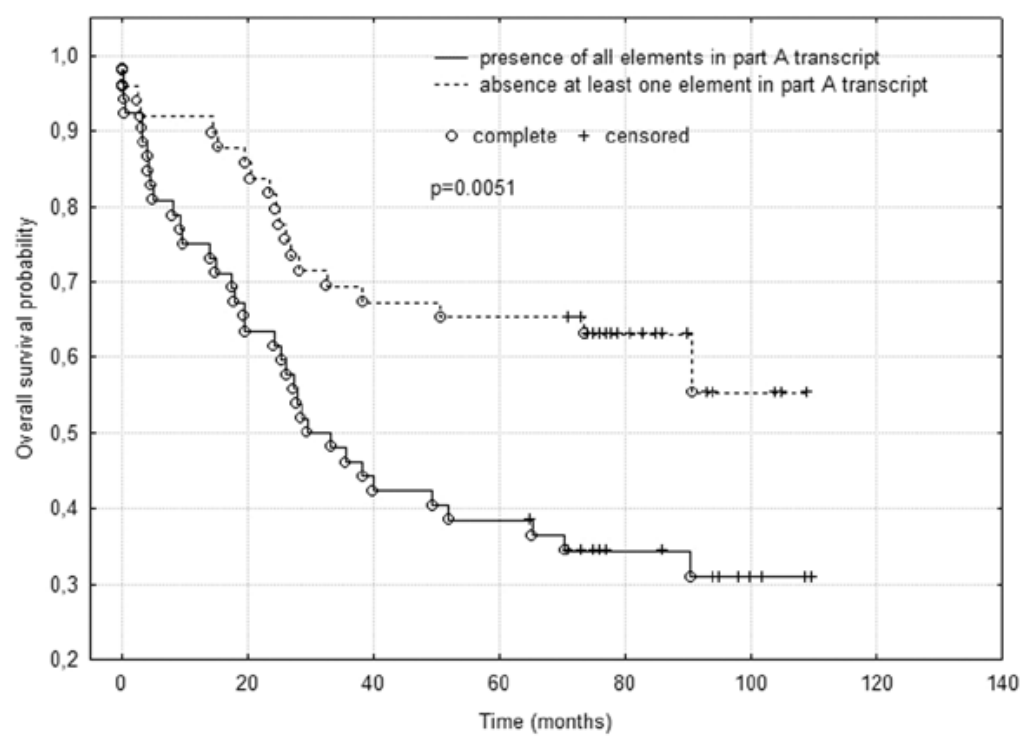

C

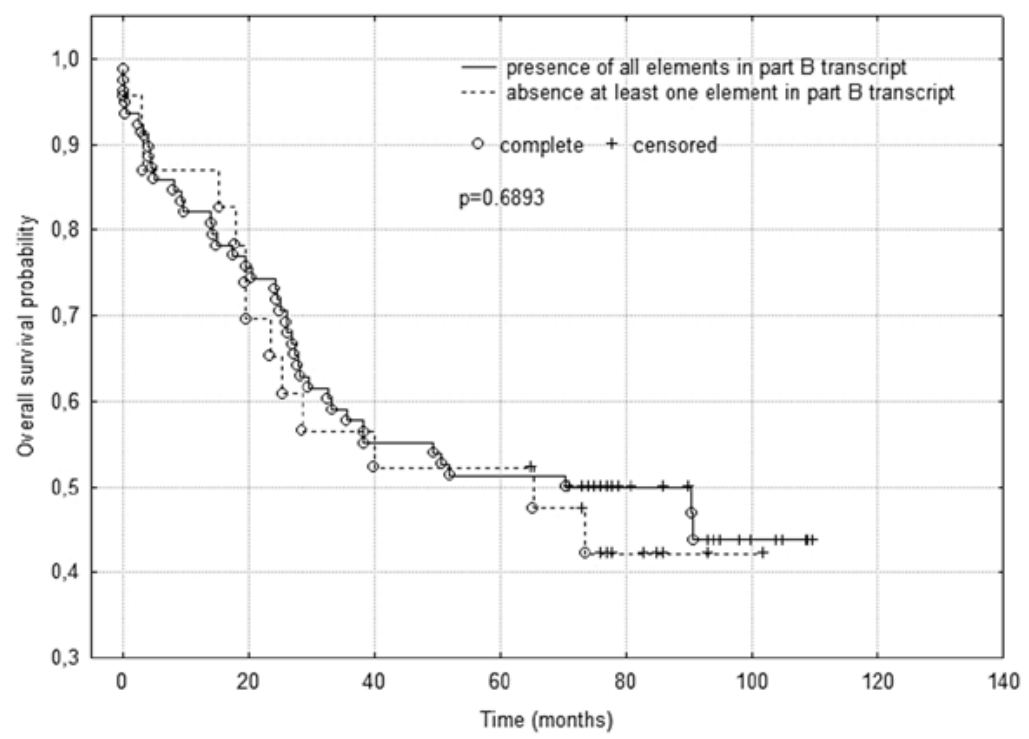

Figure 1. (A) Differences in overall survival probability in the group of patients with whole $F J 194940.1$ transcript and in the group of patients with an absence of at least one element. (B) Differences in overall survival probability in the group of patients with all elements in part A of the FJ 194940.1 gene transcript and in the group of patients with an absence of at least one element in part A of the FJ 194940.1 transcript. (C) Differences in overall survival probability in the group of patients with all elements in part B of the FJ 194940.1 transcript and in the group of patients with an absence of at least one element in part B of the FJ 194940.1 gene transcript. 
of the value of research into the association between splice variants and cancer.

The WT1 gene has four splice variants but only two of them are crucial to cancer development. These variants are termed WT1+KTS and WT1-KTS. The balance between isoforms with and without the 17-amino acid insertion appears to affect the regulation of proliferation, differentiation and apoptosis, and the prevention of Wilms' tumor formation $(14,15)$.

In light of the most up-to-date FJ 194940.1 investigations, this gene also undergoes alternative splicing, and its isoforms are present during carcinogenesis.

The FJ 194940.1 gene is localized on chromosome 1 and is physiologically expressed only in the kidney. The gene consists of 6 exons and 5 introns (1). The first intron sequence is similar to a provirus belonging to the HERVL66 family. This provirus is normally located above the FJ 194940.1 gene. It is likely that during carcinogenesis, integration of an additional copy of this virus occurs in the FJ 194940.1 gene region. This modification alters transcription in the chromosome region and the gene is then expressed in neoplastic tissue.

Establishing the nucleotide sequence of the FJ 194940.1 gene and its structure has made it possible to design primers complementary to exons and exon-exon junctions. This approach enables confirmation of the predicted exon-intron gene structure and allows us to confirm or contradict alternative splicing in this gene.

Preliminary results have shown that FJ 194940.1 undergoes alternative splicing in various tumors (4). In the preliminary experiment, the RT-PCR product for the junction of exons III/IV, and IV/V, as well as for exon III, was found in all analyzed samples (4). Products with other primers were observed irregularly (4). Findings of those studies are inconsistent with results presented in this study, in which RT-PCR products for exon $\mathrm{V}$ and the junction of exons I/II were found in all investigated cases, while other products were irregular. Lack of a RT-PCR product for exon III and junction of exons III/IV and IV/V was rare, and appeared in 12, 16 and 6 out of 102 cases, respectively. This discrepancy between the two experiments may be explained by the variable number of cases examined by the two investigators. The preliminary study counted only 30 cases, whereas in the present study 102 cases were examined.

FJ 194940.1 (previously known as P65) gene expression assessment in CRC has already been carried out $(1,16)$. At that time, the primers used were designed based on amino acid sequences. Presence of the FJ 194940.1 expression was associated with more advanced tumors with metastases to lymph nodes and distant metastases (16). These findings were confirmed by means of the real-time PCR technique. Higher levels of FJ 194940.1 expression were observed in more advanced cases, classified as III and IV according to pTNM classification (1). The results suggest that $F J 194940.1$ expression in colon cancer is engaged in the process of metastasis formation and may be associated with poor prognosis for the patient.

The presence of part A is correlated with lymphocytic tumor infiltration, which is a favorable prognostic factor. However, this factor is associated with shorter survival time. The presence of part B expression is associated with cases of low-grade malignancy, which is correlated with better prognosis for patients.
In conclusion, the investigated FJ 194940.1 gene undergoes alternative splicing. However, the role of its transcripts and potential proteins remain to be examined in further detail.

\section{Acknowledgements}

This study was supported by grant 502-03/3-015-02/50234-014 from the Medical University of Lodz, Poland.

\section{References}

1. Balcerczak E, Balcerczak M and Mirowski M: Quantitative analysis of the p65 gene expression in patients with colorectal cancer. Int J Biomed Sci 3: 287-291, 2007.

2. Balcerczak E, Bartkowiak J, Błoński JZ, Robak T and Mirowski M: Expression of gene encoding p65 oncofetal protein in acute and chronic leukemias. Neoplasma 49: 295-299, 2002.

3. Czyż W, Balcerczak E, Rudowicz M, Niewiadomska H, Pasieka Z, Kuzdak K and Mirowski M: Expression of C-ERBB2 and P65 genes and their protein products in follicular neoplasms of thyroid gland. Fol Histochem Cytobiol 41: 91-95, 2003.

4. Balcerczak E, Malewski T, Bartczak M and Mirowski M: Alternation of FJ 194940.1 transcript expression after provirus integration in human neoplasm. Int J Integrat Biol 11: 58-63, 2011.

5. Kwabi-Addo B, Ropiquet F, Giri D and Ittmann M: Alternative splicing of fibroblast growth factor receptors in human prostate cancer. Prostate 46: 163-172, 2001.

6. Chomczynski P, Sacchi N: Single-step method of RNA isolation by acid guanidinium thiocyanate-phenol-chloroform extraction. Anal Biochem 162: 156-9, 1987.

7. Orban TI and Olah E: Expression profiles of BRCA1 splice variants in asynchronous and in G1/S synchronized tumor cell lines. Biochem Biophys Res Commun 280: 32-38, 2001.

8. Baudry D, Hamelin M, Cabanis MO, Fournet JC, Tournade MF, Sarnacki S, Junien C and Jeanpierre C: WT1 splicing alterations in Wilms' tumors. Clin Cancer Res 6: 3957-3965, 2000.

9. Mixon M, Kittrell F and Medina D: Splice variant expression of CD44 in patients with breast and ovarian cancer. Oncol Rep 8: 145-151, 2001.

10. Hori M, Shimazaki J, Inagawa S, Itabashi $M$ and Hori $M$ : Alternatively spliced MDM2 transcripts in human breast cancer in relation to tumor necrosis and lymph node involvement. Pathol Int 50: 786-792, 2000.

11. Xin Y, Grace A, Gallagher MM, Curran BT, Leader MB and Kay EW: CD44v6 in gastric carcinoma: a marker of tumor progression. Appl Immunohistochem Mol Morphol 9: 138-142, 2001.

12. Aaltomaa S,Lipponen P, Ala-Opas M and Kosma VM: Expression and prognostic value of CD44 standard and variant v3 and v6 isoforms in prostate cancer. Eur Urol 39: 138-144, 2001.

13. Rys J, Kruczak A, Lackowska B, Jaszcz-Gruchała A, Brandys A, Stelmach A and Reinfuss M: The role of CD44v3 expression in female breast carcinomas. Pol J Pathol 54: 243-247, 2003.

14. Hewitt SM and Saunders GF: Differentially spliced exon 5 of the Wilms' tumor gene WT1 modifies gene function. Anticancer Res 16: 621-626, 1996.

15. Englert C, Hou X and Maheswaran S, et al: WT1 suppresses synthesis of the epidermal growth factor receptor and induces apoptosis. EMBO J 14: 4662-4675, 1995.

16. Balcerczak M, Balcerczak E, Pasz-Walczak G, Kordek R and Mirowski M: Expression of the p65 gene in patients with colorectal cancer: comparison with some histological typing, grading and clinical staging. Eur J Surg Oncol 30: 266-270, 2004. 